\title{
Télétravailler dans une collectivité territoriale : Quelle gestion des frontières entre la vie privée et la vie professionnelle?
}

\author{
Marc Dumas \\ Institut de Management de Bretagne Sud (Imabs) - Université de Bretagne Sud \\ Campus Tohannic, BP 573, 56017 Vannes Cédex
}

\section{Résumé}

Bien que le télétravail soit à un niveau faible en France, comparé à nos voisins européens, des expériences intéressantes se développent dans les collectivités territoriales qui visent à concilier des enjeux écologiques et de qualité de vie de leurs agents, mais également de modernisation du service public. Nous nous sommes intéressés au cas d'Administration qui a expérimenté le télétravail en 2009 et compte en 2013 environ 140 télétravailleurs pour un effectif de 1700 emplois administratifs et 2400 agents. Notre étude s'est focalisée sur les effets du télétravail sur la conciliation vie personnelle et vie professionnelle, ainsi que les risques d'intrusion du télétravail dans la vie privée des agents de cette collectivité.

Notre méthodologie mobilisée est double, par entretiens et par questionnaires. Après avoir conduit 25 entretiens auprès de télétravailleurs et de managers, nous avons fait passer trois questionnaires auprès de télétravailleurs, d'encadrants ainsi que de collègues de télétravailleurs. Nos résultats croisés et triangulés indiquent que le télétravail à des effets positifs tant sur le plan personnel que professionnel. II favorise la conciliation vie personnelle et vie professionnelle notamment pour les personnes connaissant le plus de difficultés. II permet de consacrer plus de temps à la famille. Les salariés ont fait preuve de capacités d'organisation et d'autonomie au travail. Alors que le télétravail pendulaire limite les effets intégratifs du travail dans la vie privée, le caractère intrusif des TIC utilisées est significatif.

(c) 2015 IDMP/Lavoisier SAS. Tous droits réservés

Mots clés : télétravail; articulation vie personnelle et vie professionnelle; fonction publique territoriale.

*Auteur correspondant : marc.dumas@univ-ubs.fr

doi :10.3166/pmp.32.107-128 @ 2015 IDMP/Lavoisier SAS. Tous droits réservés 


\section{Abstract}

Telework in a local government service : which's border management between work and private life? Although telework is at a low level in France, compared to our European neighbors, interesting experiments develop in local government service aimed at reconciling environmental issues and quality of life for their employees, but also to modernize the public service. We are interested in the case of an organization called Administration which has about 140 teleworkers in 2013 with a workforce of 1700 administrative jobs and 2400 employees. Our study focused on the effects of teleworking on reconciling personal and professional life, as well as telework intrusion risks in the private life.

Our methodology is twofold, mobilized through interviews and questionnaires. After conducting 25 interviews with teleworkers and managers, we spend three questionnaires with teleworkers, supervisors of telecommuters and teleworkers colleagues. Our results indicate that telework have positive impact on the privacy and professional life. It promotes personal life balance and career especially for people experiencing difficulties. It allows you to spend more time with the family. The employees have demonstrated organizational skills and autonomy at work. Although teleworking practice with two days a week limited integrative effects work in privacy and the intrusive nature of communication technologies use is significant.

Keywords: Teleworking, work-life issues, local government service.

\section{Introduction}

La loi de 29 février 2012 et le développement des Technologies de l'Information et de la Communication (TIC) dans les processus de travail rendent possible l'activité professionnelle de nombreux salariés en dehors des locaux de l'entreprise. Ainsi des organisations publiques et privées expérimentent et développent le télétravail pour des activités réalisées traditionnellement au bureau de manière sédentaire.

Selon l'article 46 de la loi de simplification du droit du 22 mars 2012", « le télétravail est une forme d'organisation du travail dans laquelle un travail qui aurait également pu être exécuté dans les locaux de l'employeur est effectué par un salarié hors de ces locaux de façon régulière et volontaire en utilisant les TIC dans le cadre d'un contrat de travail ou d'un avenant à celui-ci ». La loi précise des droits nouveaux aux salariés télétravailleurs (l'employeur fournit, installe et entretient les équipements...). Le décret précisant les conditions d'organisation du télétravail dans la fonction publique reste à paraître.

Le télétravail reste toutefois embryonnaire. Difficile à comptabiliser, il représente sans doute $7 \%$ des actifs, secteurs public et privé confondus (Fehrenbach, Granel, Dufort, Klein et Loyer, 2009). Le secteur public se caractérise par un retard considérable. Selon le rapport Lebreton (2013), dans la fonction publique d'État (civile, hors enseignement) un agent pour 2700 pratique le télétravail. Les établissements publics et les collectivités locales apparaissent plus entreprenants que l'administration centrale. Les collectivités territoriales

\footnotetext{
${ }^{1}$ Qui reprend la définition de l'Accord national interprofessionnel signé en 2005
} 
conduisent de nombreuses expériences (De Mazenod, 2011). Ainsi Administration ${ }^{2}$, le terrain de l'étude, a expérimenté dès 2009 le télétravail (140 télétravailleurs pour un effectif de 2400 agents). Selon une enquête de l'Assemblée des Départements de France (ADF) effectuée en 2012, 51 départements ont des projets de télétravail et de télécentres et 28 sont réellement dynamiques.

Les collectivités territoriales, qui développent le télétravail, l'inscrivent dans une démarche de développement durable (changement de mode de déplacement des agents). Les autres enjeux sont motivationnels et humains, suivront certainement les problématiques immobilières avec une gestion rationnelle des espaces de travail. Il s'agit également d'un changement culturel pour les managers de la fonction publique qui peuvent craindre de perdre le contrôle sur leurs salariés, donc l'autorité. Le télétravail peut est source d'opportunités pour d'autres, dont l'autonomie des salariés est acquise et pour lesquels c'est l'occasion de mettre des outils de suivi d'objectifs et de planification en douceur. Si le télétravail est un levier important de changement dans la fonction publique, ses effets sur l'équilibre vie personnelle et vie professionnelle, peuvent être a priori complexes. L'étude de Lefevre, Pailhé, Sollaz (2007) révèlent que les employeurs de la fonction publique d'État et hospitalière déclarent devoir souvent s'impliquer pour aider les salariés à concilier travail et famille. La réduction de l'absentéisme est particulièrement citée par les trois fonctions publiques.

Du point de vue de la littérature, le télétravail est considéré comme une pratique permettant à l'utilisateur de mieux articuler les temps personnel et professionnel, sans toutefois en avoir apporté la preuve empirique (Tremblay, Chevrier et Di Loreto, 2006). Les recherches sur l'usage des TIC tendent à montrer au contraire que les outils de communication notamment d'ordre professionnel envahissent la vie privée et tendent à brouiller les frontières (Edley, 2001).

La problématique générale de cet article est d'évaluer les effets du télétravail sur la manière dont les agents de la fonction publique territoriale articulent les différents temps sociaux : le télétravail permet-il de mieux harmoniser vie privée et vie professionnelle et si oui pourquoi? Ou au contraire favorise-t-il un brouillage des frontières? Et si oui, quels sont les risques?

L'étude porte sur le télétravail, pratiqué par les agents, mais aussi sur les TIC utilisées par les agents de la collectivité territoriale, que nous appellerons Administration. Cette collectivité territoriale se situe dans l'ouest de la France. Le département compte une population d'environ 901000 habitants (en 2012) répartis dans près de 280 communes (densité de population de 134 personnes au $\mathrm{km}^{2}$ ).

La méthodologie repose à la fois sur des entretiens auprès de membres de la Direction des Ressources Humaines, ainsi que de plusieurs autres directions et de télétravailleurs cadres et non cadres. Une première exploitation des entretiens par analyse de contenu, nous a livré des résultats qui nous ont permis de construire trois questionnaires différents qui ont été soumis aux télétravailleurs, aux encadrants de télétravailleurs et aux collègues de télétravailleur (personnes avec lesquelles les télétravailleurs travaillent régulièrement). Le croisement des données nous permet de confirmer un certain nombre de résultats issus des entretiens.

Une première partie sera consacrée à la revue de littérature et aux hypothèses issues de

${ }^{2}$ Nom d'emprunt, pour conserver l'anonymat. 
l'état de l'art. La méthodologie envisagée sera ensuite décrite. Dans une troisième partie nous présenterons les résultats que nous discuterons à la suite. Pour terminer nous mentionnerons les apports et les limites de l'étude.

\section{Cadre conceptuel et hypothèses}

Le télétravail se développe dans la fonction publique. Outil de développement durable et de motivation, il peut contribuer à la performance des agents en favorisant le bien-être et notamment une meilleure articulation des temps sociaux.

\subsection{Le télétravail dans la fonction publique territoriale}

Le développement des TIC et la généralisation de la couverture du haut débit en France ont rendu possible de nouvelles formes d'organisation, telles que le télétravail formel ${ }^{3}$. « La dématérialisation démultiplie les possibilités de travailler en dehors du cadre bureaucratique classique : travailler sur plusieurs sites d'une même administration, à l'extérieur (réunions, conférences, séminaires) à domicile ou dehors (dans la rue ou en déplacement) » ${ }^{4}$. Pour des administrations publiques, et en particulier les collectivités locales, l'exemplarité en matière de développement durable a conduit certaines grandes administrations, telles que des Conseils généraux à intégrer des projets de télétravail dans leurs politiques environnementales et les agendas $21^{5}$. L'objectif est notamment de réduire l'usage de la voiture individuelle et de promouvoir d'autres modes de déplacement.

Comme l'écrivent les auteurs de la note d'analyse Travail emploi du Centre d'analyse stratégique, «à bien des égards les collectivités apparaissent comme des entités plus dynamiques et plus ouvertes aux TIC que la fonction publique d'État alors que moins de $20 \%$ des fonctionnaires territoriaux ont un poste compatible avec du télétravail ». Ils notent également que « l'implication des décideurs politiques et manager en faveur de la responsabilité sociale des administrations est très forte. Ainsi les motivations mises en avant le plus souvent sont le développement durable et l'amélioration du cadre de vie des personnels. Ces deux objectifs, qui répondent à des aspirations communes à leurs agents, recueillent un consensus en interne et contribuent à la qualité de vie au travail des fonctionnaires territoriaux ».

La contrainte budgétaire marque fortement le contexte des collectivités ces dernières années. La maîtrise de la masse salariale et la rareté financière obligent à se poser des questions en termes d'organisation à adopter, de performance, de motivation des agents et de mutualisation des compétences. Ceci induit un changement de culture. Le télétravail peut apporter des solutions. Il participe à la modernisation du service public de par la nécessité de passer au management

\footnotetext{
${ }^{3}$ À distinguer du télétravail informel qui se fait en débordement généralement à domicile ou en déplacement hors temps de travail légal.

${ }^{4}$ "Quel est l'impact des TIC sur les conditions de travail dans la fonction publique? Centre d'analyse stratégique, LA NOTE D'ANALYSE Travail Emploi, janvier 2013, nº 318.

${ }^{5}$ L'Agenda 21 (ou Action 21) est comme l'indique son nom, un plan d'action pour le XXle siècle. Les collectivités territoriales sont appelées, dans le cadre du chapitre 28 de l'Agenda 21 de Rio, à mettre en place un programme d'Agenda 21 à leur échelle, intégrant les principes du développement durable, à partir d'un «mécanisme de consultation de la population ».
} 
par objectifs. Il permettrait de booster le développement d'une culture de l'évaluation et de résultats par objectifs. Le télétravail est supposé apporter de la flexibilité aux agents dans la gestion des différentes contraintes personnelles et professionnelles, en leur permettant d'avoir plus de temps pour soi ou pour les autres, de passer plus de temps auprès de sa famille et ses enfants, d'avoir plus de disponibilité, de souplesse, pour se rendre à des rendez-vous chez des médecins. Il peut être une solution d'amélioration de la qualité de vie au travail pour des agents des services publics soumis à la pression d'un public de plus en plus exigeant.

Le télétravail est une forme d'organisation spatio-temporelle, qui peut être effectuée depuis le domicile ou un télécentre, plus proche de son domicile que le lieu de travail habituel. L'intérêt de cette dernière forme d'organisation du télétravail est de préserver la séparation domicile lieu de travail tout en diminuant les temps de transport. La notion de télétravail pendulaire ou alternant signifie que le télétravailleur partage son temps de travail entre des lieux fixes : le domicile ou le télécentre et le lieu habituel de travail (Morel à l'Huissier, Turbé-Suetens, 2012).

\subsection{Le télétravail favorise-t-il la conciliation vie personnelle et vie professionnelle?}

Le télétravail a souvent été associé à une amélioration de l'équilibre entre le travail et la vie personnelle (Madsen, 2003). Vivre une vie équilibrée, c'est vivre des expériences satisfaisantes dans tous les domaines de sa vie, avec des ressources personnelles (l'énergie, le temps et l'implication) bien réparties entre les différentes sphères (Dumas, 2008a). Plus l'individu disposera de ressources personnelles à consacrer à un rôle et plus l'équilibre sera dit positif (Othman, Yusef et Osman, 2009). Il peut être également déterminé par le niveau de satisfaction au travail et dans la vie personnelle et familiale, par la flexibilité du temps de travail (la durée et les horaires de travail) ainsi que la flexibilité du lieu de travail (choisir son lieu de travail, en travaillant chez soi ou à proximité).

Le sentiment d'équilibre entre les sphères privées et professionnelles peut être facilité quand les personnes ont suffisamment de temps pour remplir des activités dans les contextes professionnels et privés. Le modèle de Karasek (1979) peut en être une illustration : quand le contrôle sur une exigence du travail augmente, le conflit entre le travail et la vie privée diminue et l'équilibre devient négatif. Brey (1999) montre que le télétravail accroît l'autonomie des personnes. En effet, la capacité à contrôler le temps de travail est essentielle dans la gestion de multiples exigences/rôles, et l'opportunité de travailler au domicile permet aux personnes d'exercer les prescriptions familiales avec un meilleur état d'esprit (Hill, Hawkins, Ferris et Weitzman, 2001). Golden et Veiga (2005) ont par ailleurs montré que l'autonomie dans l'emploi et la flexibilité des horaires de travail exercent un effet modérateur du télétravail sur le conflit travail-famille.

Les avantages du télétravail pour l'équilibre entre les vies personnelle et professionnelle ont été mis en évidence par une méta-analyse recensant 46 études couvrant 12883 employés (Gajendran et Harrison, 2007). Un des facteurs explicatifs majeurs résiderait dans la flexibilité temporelle (Tremblay, Chevrier et Di Loreto, 2006) autorisant notamment la prise de rendez-vous dans la journée, source de diminution du stress relatif à la gestion de rôles multiples (professionnels et parentaux). Ces résultats sont à confirmer sur un échantillon d'agents territoriaux français.

Ceci nous amène à formuler une première hypothèse : le télétravail favorise l'équi- 


\section{libre vie personnelle et vie professionnelle de l'agent en lui apportant une meilleure flexibilité dans leur vie au quotidien.}

\subsection{Le télétravail peut brouiller les frontières et conduire à un travail sans fin}

Plusieurs études sur le télétravail s'interrogent sur la perméabilité des frontières entre la vie personnelle et professionnelle (Dumas, Ruiller, 2014) et le degré d'empiétement d'une sphère sur l'autre (lieu et temps) avec pour conséquences le développement d'émotions négatives (irritabilité, solitude, anxiété, culpabilité) pour certaines personnes associées à un travail sans fin ou à une difficulté à stopper le travail (Baines et Gelder, 2003; Mann et Holdsworth, 2003). Ainsi des salariés peuvent également présenter des addictions par manque de séparation entre le travail et la vie privée (Baines et Gelder, 2003).

Selon l'étude de Mustafa (2012), les télétravailleurs à domicile peuvent vivre un sentiment de déséquilibre en tentant de gérer les exigences de leur travail à côté des attentes de leur famille. Des répondants signalent que du fait de leur présence au domicile, leur famille a de très fortes attentes à leur égard. Quinze des vingt répondants se plaignent que leur environnement familial ou proche ne perçoit pas leur travail comme un véritable travail. Selon Tremblay, Chevrier et Di Loreto (2006, p. 4), «les membres de l'entourage ne comprennent pas toujours les limites du télétravailleur et se permettent de formuler des demandes de disponibilité qu'ils ne formuleraient pas si la personne ne travaillait pas à la maison». À l'inverse «pour l'entourage, les parents, les amis, le fait de voir le télétravailleur travailler quelques heures le week-end peut les inciter à dire que celui-ci est toujours en train de travailler ». Ainsi, le télétravail peut être source de conflits familiaux (Haddon et Lewis, 1994 ; confirmant les résultats de Christensen, 1987). Cependant, Tremblay $(2002,2003)$ ainsi que Felstead et Jewson (2000) ne font état que de problèmes mineurs d'adaptation au début de l'installation à la maison. Les télétravailleurs établiraient des règles de fonctionnement avec les membres de leur famille et cela ne créerait pas de friction au sein de la famille (Tremblay, Chevrier et Di Loreto, 2006).

Pour maintenir une frontière entre le travail et sa vie privée, Kowalski et Swanson (2005) suggèrent que le télétravailleur adopte des stratégies telles que définir un espace spécifique pour le travail au domicile, établir des rituels matinaux au foyer afin de marquer la journée de travail (par exemple s'habiller comme s'il allait au bureau), informer ses enfants et amis qu'il télétravaille et qu'il ne peut être dérangé durant les heures de travail. Kossek et al. (2006) établissent aussi que les salariés doivent avoir une gestion formelle des frontières pour organiser et séparer les exigences du travail et les attentes spécifiques provenant du domaine familial.

Les effets du télétravail peuvent dépendre du degré de séparation ou de segmentation/ intégration des sphères (cognitives, physiques, comportementales et communicationnelles) (Kreiner, 2006). Les recherches de Nippert-Eng (1996) ont montré que certains individus sont principalement intégrateurs et aiment mélanger les rôles professionnels et extraprofessionnels. À l'inverse, certains sont séparateurs et préfèrent séparer le travail et la vie privée. Les individus ayant un désir élevé de segmentation seront plus attirés par les pratiques basées sur la séparation des rôles telles que les horaires flexibles, plutôt que le télétravail, propice à l'intégration (Matthews et al., 2010).

Une étude récente de Bourhis et Mekkaoui (2010) modère ces derniers résultats signalant 
que le télétravail ne peut être perçu par les personnes comme une stratégie d'intégration mais au contraire comme une pratique favorisant la flexibilité des frontières spatiales et temporelles (Rau et Hyland, 2002). Aussi, le télétravail n'étant que de quelques jours et jamais à temps plein (Golden et Veiga, 2005 ; Schweitzer et Duxbury, 2006), les salariés en télétravail occasionnel ne modifient pas leur mode de garde d'enfants, par exemple. Par ailleurs, l'employeur ou le manager peuvent convenir de règles avec les salariés afin que leur vie privée n'interfêre pas avec le travail (ne pas télétravailler en retour de congé; dissocier les jours de télétravail et de RTT...).

La notion d'intrusion a été approfondie par Kreiner (2006), qui explique qu'elle survient quand l'individu désire segmenter mais que « la violation » de la frontière le force à l'intégration. À l'inverse quand un individu désire l'intégration mais que la segmentation est forcée, la distanciation peut être mal vécue. Chaque télétravailleur développe donc des stratégies pour gérer la frontière entre la «distance » et « l'intrusion » par une utilisation adaptée des TIC (Kreiner, Hollensbe et Sheep, 2009)

En situation de télétravail, les TIC permettent de garder le contact avec l'équipe et le manager et d'éviter l'isolement. Des habitudes ou rituels tels que dire bonjour et au revoir aux collègues bornent généralement une journée de travail alors qu'elle n'est pas « contrôlée par une pointeuse ou par la présence physique d'un chef, lorsque les collègues ne sont pas là pour définir une norme temporelle collective? » (Rey et Sitnikoff, 2006, p. 4). Échanger un document partagé, communiquer en conférence téléphonique ou via un outil similaire à MSN sont indispensables au maintien d'une communication efficace et ces outils permettent également de faire face à des imprévus tels que des réunions non planifiées et garantir une présence virtuelle à la réunion.

Si les outils peuvent favoriser le débordement d'une sphère vers l'autre, le travail sans fin, le télétravailleur peut à l'inverse utiliser les technologies pour gérer les frontières de sa vie professionnelle et de sa vie personnelle (Fernandez, Guillot, Marrauld, 2014). D'autres vont pouvoir développer des usages des TIC pour les aider à séparer leur domaine de vie : avoir deux téléphones portables, un professionnel et un à usage personnel, avoir deux adresses mail, une professionnelle et une autre personnelle... (Clark, 2000).

Ceci nous conduit à formuler une seconde hypothèse double :

2.1. Le télétravail favorise l'intrusion du travail dans la vie privée, l'intégration plutôt que la flexibilité des frontières, du fait des TIC.

2.2. Le télétravail ne favorise pas l'intrusion du travail dans la vie privée, s'il s'accompagne d'une bonne maîtrise des TIC.

\section{Méthodologie}

Le télétravail chez Administration a démarré à titre expérimental en octobre 2009 avec 27 agents volontaires. La distance domicile travail constituait le principal critère de sélection des candidatures. L'expérimentation a été étendue à 9 directions volontaires en septembre 2010. La généralisation aux autres directions s'est faite à partir de janvier 2012 (encadré 1). Le télétravail constitue une modalité de l'organisation du temps de travail pour les agents administratifs (1700 personnes). À la date de l'étude, Administration compte environ 140 télétravailleurs représentant les différents métiers administratifs de la collectivité territoriale. 


\section{Encadré 1 : Les étapes de la mise en place du télétravail chez Administration}

Mai à décembre 2008 : Étude de faisabilité, consistant à mener une enquête le plus large possible en France et en Europe, sur des expérimentations existantes de télétravail et ainsi en étudier la possible adaptation au sein de la collectivité. Suite à la remise du rapport d'étude au président du Conseil général, la mise en place d'une expérimentation a été décidée.

Mars - octobre 2009-juin 2010 : Une équipe de 4 personnes a construit l'ossature du projet qui a démarré par une phase d'expérimentation dès octobre 2009. Vingt-sept agents volontaires sur un échantillon représentatif des différentes directions, toutes catégories et métiers confondus, ont participé à cette expérimentation. Le télétravail s'organise sur une durée de deux à trois jours maximum avec trois dispositifs possibles : à domicile; en télécentre dépendant d'Administration; en télécentre partenaire. Chaque télétravailleur a signé un contrat d'engagement avec son encadrement, qui détaille avec précision les activités pouvant être réalisées en télétravail, les plages de disponibilité et les horaires de travail, les jours télétravaillés. Un guide d'auto-évaluation et une charte du télétravail ont été établis. Le premier aide le manager et le télétravailleur à se situer face à un travail à distance, et le second précise les droits et les devoirs des télétravailleurs pendant la phase d'expérimentation. Des rencontres régulières ont eu lieu avec les organisations syndicales. Après un bilan positif en juin 2010, l'expérimentation est étendue.

Octobre 2010 - septembre 2011 : Deuxième phase expérimentale. Neuf directions se sont portées volontaires pour participer à l'extension de l'expérimentation. 60 personnes sur un effectif de 250 personnes se sont déclaré candidates; ce qui porte le nombre de télétravailleurs à environ 80 à 90 personnes. Les règles sont assouplies pour ce qui concerne le temps télétravaillé qui s'articule désormais en fonction de la mission et de l'organisation du service. Les trois possibilités de lieux de télétravail restent identiques. Un PC portable est mis à disposition du télétravailleur à domicile. Les agents en télécentre disposent d'un bureau avec un poste fixe dédié.

Cette phase d'extension de l'expérimentation doit permettre de mettre en œuvre et de vérifier les conditions d'une généralisation du télétravail à compter de janvier 2012.

Plusieurs enquêtes et restitutions ont été effectuées pour accompagner sa mise en place : un questionnaire pour le bilan intermédiaire en juillet 2009, suivi d'une réunion en février 2010; un bilan de l'expérimentation en mai 2010. L'étude la plus récente, qui fait l'objet de cet article, a été menée en deux temps.

\section{L'étude par entretiens}

Entre le 10 mai et le 3 juillet 2012, vingt-trois entretiens ont été menés et au total vingtcinq personnes ont été interrogées, dont vingt télétravailleurs, soit quatorze femmes et six hommes (cf. annexe I). La durée des entretiens varie de quarante-cinq minutes à deux heures. Les entretiens ont été retranscrits intégralement et ont fait l'objet d'une analyse de contenu manuelle par thématique, structurée en fonction des thèmes du guide.

Parmi les vingt télétravailleurs, cinq ont expérimenté le télétravail, et télétravaillent donc depuis octobre 2009. Nous avons également rencontré dix managers de télétravailleurs dont cinq 
télétravaillent. La distance domicile travail peut constituer un motif d'adoption du télétravail pour quatorze agents qui résident à trente kilomètres et plus de leur lieu de travail. Trois télétravailleurs sont domiciliés à moins de dix kilomètres de leur lieu de travail et ont choisi le télétravail pour expérimenter (deux femmes ont été incitées par leur supérieur hiérarchique également chef de projet télétravail) ou encore par attrait pour cette nouvelle forme d'organisation.

\section{L'étude par questionnaires}

Pour la suite de l'étude, nous avons envisagé d'élaborer et de diffuser un questionnaire, qui a été motivé par trois raisons principales : premièrement, nous souhaitions offrir la possibilité à tous les télétravailleurs de s'exprimer. Le taux de réponse est d'environ $95 \%$ (132/138) et de $76 \%$ pour les questionnaires exploités (105/138). Deuxièmement, n'ayant pas interrogé d'agents non-télétravailleurs hormis l'encadrement en phase d'entretiens, nous étions en attente d'informations sur leur ressenti vis-à-vis de cette organisation du travail. Troisièmement, nous voulions recueillir des éléments de réponse d'un nombre plus conséquent de cadres, d'encadrants, de télétravailleurs. Nous avons élaboré les questionnaires en concertation avec le responsable de mission, qui a participé à la construction des questionnaires précédents. Ils ont été validés et testés par une équipe de 6 personnes, composée pour moitié d'encadrants.

Ces trois questionnaires (annexe II) ont été présentés avec le logiciel Lime Survey et complétés en ligne. La chargée de projet télétravail était responsable de leur diffusion auprès des cibles concernées. En mai, un courrier a été adressé aux directeurs les informant du déroulement de l'enquête. Les télétravailleurs et leurs responsables ont reçu l'adresse internet des questionnaires respectifs début juin. Il a été demandé aux encadrants d'informer les collègues des télétravailleurs, qu'ils doivent répondre au questionnaire mis à leur disposition sur l'intranet. Signalons qu'il n'était pas demandé aux encadrants d'inciter les télétravailleurs à répondre.

L'enquête s'est donc déroulée sur moins d'un mois du 4 juin au 29 juin (lancement le 4 juin; relance le 14 juin; seconde relance le 21 juin). Les retours sont satisfaisants puisque 132 télétravailleurs, 40 managers et 61 collègues de télétravailleurs ont répondu. Les questionnaires complets exploités sont respectivement de 105, 35 et 51 (cf. annexe III). Le très bon taux de retour des questionnaires des télétravailleurs s'explique par la confiance envers la chargée de mission, la communication des résultats à l'issue de la phase d'enquête par entretiens et la suppression de questions d'ordre privée (nombre d'enfants, situation familiale) pour préserver l'anonymat.

\section{Résultats}

Nous présentons les résultats en abordant successivement pour chaque hypothèse les synthèses issues de l'analyse de contenu, puis celles issues de l'exploitation des questionnaires. Les résultats issus des questionnaires confirment et affinent ceux des entretiens.

\subsection{Le télétravail, un outil efficace d'harmonisation vie privée et vie professionnelle?}

Les entretiens menés auprès des télétravailleurs indiquent que la souplesse temporelle du télétravail a pu entraîner des changements variés dans la vie des personnes et pour des raisons différentes (matériel, financier...) améliorer leur vie. Quatre récits l'illustrent. 
Le télétravail a permis à un jeune informaticien de gagner en souplesse et de changer de mode de transport en commun. Il prend désormais le bus. Il nous confie : "C'est moins cher que le train. Il y a un aspect coût financier. Je sais qu'en étant télétravailleur, si j'ai des coups de bourre je peux continuer chez moi. »

Une assistante qui connaît des problèmes de santé a été jugée apte au travail sous condition de télétravailler (trajet domicile-travail de soixante kilomètres). Par ailleurs le télétravail lui permet de suivre ses séances de kinésithérapie en milieu de journée. «Avec mon handicap j'ai des rendez-vous chez le kiné toutes les semaines. Il est débordé entre 17 et 19 heures. J'ai mon rendez-vous entre 13 heures et 13 h 30 le vendredi toutes les semaines, avec les horaires variables c'est possible ». Pour un autre agent, le télétravail lui permet de se rendre à des rendez-vous médicaux : «Cela m'évitait de prendre une demi-journée de RTT».

Un directeur adjoint a fait le choix de télétravailler pour des raisons de famille. « Je me suis rendu compte que j'étais rarement avec les enfants. Je suis présent pour des moments clés et essentiels pour la vie de mes enfants ». Toutefois il sent une difficulté à maintenir cette journée de télétravail par semaine : "on peut changer mais c'est difficile de trouver une journée disponible sans rendez-vous...».

Ces quatre agents apprécient le télétravail car il leur permet notamment de mieux gérer leur temps ou de passer plus de temps en famille. Notons que peu d'agents ont exprimé des difficultés d'ordre familial ou de contraintes personnelles pour motiver leur candidature au télétravail. Si elles existent, elles sont également associées à un temps de trajet long (40 à $60 \mathrm{~km}$ ). Ainsi une mère de famille divorcée avec deux adolescents cumule charge familiale et un temps de trajet de 40 kilomètres aller en voiture; Une autre cumule un trajet aller de 60 kilomètres et un bébé qu'elle élève seule (conjoint militaire régulièrement en déplacement).

Pour affiner la compréhension de l'usage du télétravail, nous avons intégré dans le questionnaire à l'intention des télétravailleurs plusieurs questions qui abordent l'articulation des temps de vie; la vie de famille et les aménagements horaires personnels rendus possibles par le télétravail.

S'agissant du premier thème, l'articulation des temps de vie, le télétravail a permis de passer plus de temps en famille pour près de $70 \%$ des télétravailleurs, soit près de $85 \%$ des salariés concernés (14,3\% se disent pas concernés). Le télétravail facilite le mode de garde des enfants pour $26,7 \%$ des télétravailleurs (soit 58,3\% des salariés concernés). En outre il permet de s'accorder plus de temps pour soi pour 70,3\% des télétravailleurs, de faire moins de courses et de tâches ménagères le week-end pour 43,8\% des télétravailleurs (annexe VI).

Un second thème concerne exclusivement la sphère familiale et tend à approfondir les résultats précédents. Globalement le télétravail est jugé avoir un effet positif pour la famille sur l'ensemble des items proposés (annexe VII). Environ 70 \% des télétravailleurs concernés estiment que le télétravail a un effet positif sur la famille en permettant de passer plus de temps avec les enfants ainsi que de passer plus de temps avec le conjoint, tandis que plus de la moitié des télétravailleurs jugent que le télétravail permet un partage des tâches dans le couple. Ce sentiment est partagé autant par les hommes que par les femmes. Un tiers des télétravailleurs concernés répondent que le télétravail permet de passer plus de temps auprès des personnes âgées, notamment des parents (annexe VII).

Enfin pour le troisième thème, nous avons souhaité évaluer l'importance des aménagements horaires personnels. Ainsi $26 \%$ des télétravailleurs ont adapté leur temps de travail en dehors des plages de disponibilité défini dans le contrat d'engagement (cadres : 27,9\% ; 
non-cadres : 24,2 \%; hommes : 27,9\%; femmes : 24,2 \%). Le télétravail semble apporter une flexibilité dans les horaires de travail pour un quart des télétravailleurs qui ont aménagé leur temps de travail en dehors des plages horaires définies dans le contrat d'engagement.

Ces résultats attestent que le télétravail permet réellement de passer plus de temps avec la famille, les proches, les enfants et le conjoint et est efficace pour résoudre des problèmes de conciliation vie personnelle et vie professionnelle. Le télétravail est un outil de conciliation plus égalitaire.

Ces résultats valident l'hypothèse 1 : le télétravail favorise la conciliation vie personnelle et vie professionnelle

\subsection{Brouillage des frontières et quel usage des TIC?}

Plusieurs entretiens confirment la littérature sur la mise en garde d'un risque de brouillage des frontières, notamment qu'un des risques du télétravail en particulier à domicile est d'être mis plus facilement à contribution par leur famille. Ainsi ce père a pu s'occuper de ses deux enfants durant la grève des cantines dans sa ville. "Une grève de cantine a duré un mois, je pouvais aller les récupérer 2 jours par semaine ». Plusieurs personnes évoquent la présence de membres de la famille au déjeuner et des interruptions pour préparer le repas : «En télétravail, je fais des pauses. Il peut m'arriver de mettre quelque chose à cuire...je mange avec mon mari. Je ne ressens pas le besoin de m'arrêter en milieu de matinée ». Autre illustration pour cet homme qui prépare le repas pour sa femme qui rentre à treize heures et qui répond peut-être à une sollicitation familiale.

Les télétravailleurs sont conscients de ces risques et les qualités requises pour le télétravail, qu'ils évoquent sont : être autonome, être vigilant et ne pas se laisser distraire, ne pas se disperser. Un agent confie : "Il faut se dire que le télétravail est véritablement du travail et n'est pas une rupture dans sa semaine de son activité professionnelle. Il faut rester performant.»

Deux facteurs nous paraissent limiter les risques d'intrusion. Premièrement, les règles qui encadrent le télétravail (règles définies dans un cadre collectif : $50 \%$ de son temps de travail au maximum, rythme hebdomadaire, jours fixes, règles de réversibilité et de flexibilité) limitent les effets intégratifs durables du professionnel dans la vie privée. Deuxièmement, l'état d'esprit du télétravailleur, sa volonté et sa capacité à segmenter les sphères privée et professionnelle vont contenir ce chevauchement ou ce brouillage des frontières. Ainsi, selon une directrice, « certains veulent une coupure, séparer les choses; ont peur de ne pas s'organiser, de se faire submerger...». Cette capacité à organiser son temps (ses horaires) et ses tâches en séparant ce qui est du professionnel et du personnel va permettre d'éviter cette intégration des activités professionnelles et privées réalisées dans une unité de lieu. Ainsi une assistante atteste que le télétravail «nécessite une grosse organisation, de se dire je vais faire cela. Cela permet d'avoir une vision globale de son travail. ... J'ai une pochette à rabats, je mets des choses dans cette pochette télétravail qui va aller avec la sacoche de l'ordinateur portable. Ça m'a permis de m'organiser ». Le travail d'analyse et de définition des tâches réalisées en télétravail est fait sous le contrôle du chef de service et est un préalable au télétravail.

Nos résultats démontrent que l'usage des TIC va accroître la porosité entre la vie professionnelle et la vie personnelle. Tout d'abord l'usage de la ligne téléphonique personnelle pour recevoir les appels au domicile est mal ressenti par les télétravailleurs. Selon un 
directeur, «les télétravailleurs disent qu'il y a débordement du travail vers la vie privée car ils doivent utiliser leur ligne téléphonique ». Toujours selon lui les critiques sur le téléphone concernent « le $n^{\circ}$ apparent, la ligne bloquée et le fait de tomber sur le répondeur familial». Certains appels reçus à domicile peuvent déranger et inquiéter. Ainsi selon les assistantes de la Direction Générale (DG) « avec l'enquête Qualité de Vie au Travail (début 2011), des gens en souffrance appelaient la DG pour avoir des rendez-vous. Le fait de les recevoir à la maison, ça perturbait, ça touche à l'intimité, le fait que le numéro apparaît... On ne peut pas en discuter avec sa collègue. On est un peu isolé. Ça a duré une période».

Par ailleurs des solutions existent et ont été mises en œuvre par la grande majorité des agents. Certains télétravailleurs ont trouvé des parades en recourant à l'usage du numéro ADSL (09) pour le renvoi des appels professionnels. D'autres ont fait le choix d'un téléphone portable dédié. Cette responsable de cellule a «fait le choix de dédier un téléphone portable au professionnel car je n'avais pas envie qu'on voit mon numéro de fixe ». Des assistantes de la DG utilisent également un téléphone portable. Elles font un transfert la veille sur le portable et un renvoi le matin sur le fixe. Les deux utilisent le portable pour appeler un portable : «On a un téléphone portable personnel. Les appels sont renvoyés dessus ». D'autres personnes ne rappellent que les téléphones fixes car les appels aux portables ne sont pas compris dans leur forfait.

Nous observons deux types de comportement : les séparateurs et les intégrateurs. Les usages des TIC associés au télétravail ont permis au salarié une meilleure maîtrise de leurs horaires de travail du fait de la possibilité de poursuivre des tâches au domicile. Le débordement a remplacé les horaires tardifs ponctuels. À l'inverse certains privilégient une segmentation. Ainsi une assistante de direction explique : "J'ai une grande sacoche dans laquelle j'ai un micro-ordinateur portable et un téléphone portable que j'allume uniquement le mercredi et le vendredi. L'ordinateur portable ne sert qu'au travail. Jamais il ne me serait venu de prendre l'ordinateur du télétravail. J'emprunte celui de ma saur si j'en ai besoin. Je veux séparer le professionnel du personnel. Le week-end je l'ai chez. moi et je ne l'utilise pas ».

Alors que les entretiens nous ont permis d'identifier trois facteurs de risque d'intégration : les sollicitations par la famille; les horaires tardifs ; les réponses aux messages hors temps de travail, nous avons souhaité confirmer ces premiers résultats. Ainsi nous poursuivons avec les données issues des réponses aux questionnaires qui se rapportent à trois thèmes principaux : la séparation entre vie personnelle et vie professionnelle, l'usage des moyens de communication et le choix du lieu de télétravail.

Concernant le premier thème, le télétravail ne pose pas de problème de séparation entre vie personnelle et vie professionnelle du fait de sollicitations par la famille pour $97,1 \%$ des télétravailleurs. $17,2 \%$ estiment que le télétravail peut poser des problèmes de séparation du fait d'horaires tardifs. Ce résultat signifie que le risque de travail sans fin est peu présent. Enfin à l'exception d'une personne (un homme cadre), tous disent que pendant le(s) jour(s) télétravaillé(s), ils parviennent à séparer les temps de vie professionnelle et personnelle (annexe 4). À l'inverse 25,7 \% estiment que le télétravail pose des problèmes de conciliation du fait de réponses aux messages hors temps de travail. La présence d'un ordinateur professionnel à domicile conduit à répondre à des messages hors temps de travail. Par ailleurs, les cadres se distinguent nettement des non-cadres en reconnaissant que le télétravail sur les différents points mentionnés pose davantage de problèmes de séparation 
entre la vie professionnelle et la vie personnelle (annexe 5). Le risque de travail sans fin, soulevé par des cadres, ne semble pas une crainte partagée par les non-cadres.

S'agissant des moyens de communication utilisés, le principal facteur d'intrusion identifié lors des entretiens est confirmé par l'étude par questionnaires. En effet, 28,6\% des télétravailleurs estiment que les appels téléphoniques sur un poste fixe posent un problème de séparation. Les appels téléphoniques en dehors des horaires de travail posent des problèmes de séparation pour 13,3\% des télétravailleurs. Les résultats confirment que le téléphone portable personnel est l'outil personnel le plus utilisé pour un usage professionnel les jours de télétravail, et 50,5\% des télétravailleurs y ont recours. Les autres TIC personnelles (clavier, écran, souris) sont utilisées par un quart à un tiers des télétravailleurs.

Le troisième thème aborde le lieu de télétravail et les facteurs qui influencent son choix. Alors que près de $85 \%$ des télétravailleurs (89) télétravaillent à domicile, plus de la moitié d'entre eux (62\% soit 55) ne souhaitent pour aucune raison passer en télécentre $(61,8 \%)$. $27 \%$ (24) choisiraient le télécentre s'il y en avait un à proximité de leur domicile; 10,1\% (9) feraient le choix du télécentre pour rompre avec l'isolement et 12,3\% (11) pour des raisons de séparation vie privée et vie professionnelle. Lorsque l'on interroge les 16 télétravailleurs en télécentre, ils sont 43,75\% (7) à ne pas souhaiter passer en télétravail à domicile; 18,75\% (3) choisiraient le domicile s'ils avaient un espace bureau adapté au domicile; enfin 37,5\% (6) passeraient en l'absence de personnes à domicile durant les horaires de travail. L'hypothèse 2.1 est partiellement validée, alors que 1'hypothèse 2.2 est confirmée. Hypothèse 2.1 : Le télétravail peut brouiller les frontières et conduire à un travail sans fin. Hypothèse 2.2 : Le manque de maturité à l'égard des TIC et le manque d'outils de communication adaptés favorisent une intrusion du télétravail dans la vie privée des agents (dans le cas d'un télétravail à domicile).

\section{Discussion}

L'étude confirme que le télétravail favorise la conciliation des temps en apportant aux agents une flexibilité temporelle qui leur permet de mener leurs activités personnelles dans de meilleures conditions et de consacrer plus de temps à leur famille sans nuire au travail, mais en adoptant une organisation du travail transparente pour l'institution.

Nous notons une similitude entre les discours recueillis et l'exploitation des questionnaires aussi bien des télétravailleurs que des encadrants. Les résultats sont cohérents avec les objectifs et la lecture qu'en a la direction. Ainsi pour un directeur adjoint, le télétravail est un outil et peut être une réponse pour des personnes qui ont envie de vivre mieux, mieux concilier vie personnelle et vie professionnelle, réduire son temps de trajet. Plusieurs directeurs évoquent l'intérêt du télétravail alors qu'ils n'ont "plus de levier positif vis-àvis des agents (rémunération, carrière...) ». "On peut faire plaisir à des personnes mais pas à quelles conditions... avant de partir en télétravail il faut voir quelles tâches sont télétravaillées et du coup on peut tirer sur la ficelle de l'organisation. Si on avait tiré sur cette ficelle, les agents auraient résisté... Le télétravail apporte une dynamique positive».

Trois résultats principaux se dégagent de notre étude : la meilleure conciliation vie personnelle et vie professionnelle, les risques d'intrusion et les capacités d'adaptation et d'autonomie des agents. 
Premièrement, le télétravail a permis de réduire les temps de transport et de s'accorder plus de temps pour soi et/ou pour la famille, pour les tâches domestiques et logistiques à l'heure de midi ou en fin d'après-midi alors qu'elles étaient réalisées le week-end. Plus de temps pour la famille, c'est aussi un meilleur partage des tâches au sein des couples, notamment pour les hommes et plus de temps passé auprès des parents. Le télétravail offre aux agents d'Administration une souplesse des horaires de travail, sans avoir à poser de RTT ou à solliciter une autorisation d'absence de son manager. Le télétravail est ici associé à une plus grande liberté de gestion du temps de travail. Le télétravail «souple » (liberté du rythme, du jour) conjugué aux horaires variables constitue un avantage dont profitent en particulier les cadres d'Administration et qui est aujourd'hui possible pour tous les télétravailleurs. Le télétravail est bel et bien un outil de qualité de vie au travail et est un levier de performance.

Par ailleurs, le télétravail peut également être une organisation perçue comme ergonomique pour des personnes en difficultés (famille monoparentale ou conjoint souvent absent, parent divorcé, suivi médical...) en apportant une souplesse dans l'organisation des déplacements nécessaires et en redéfinissant les frontières entre le travail et le « hors travail ».

Deuxièmement, si le télétravail facilite la conciliation des vies personnelle et professionnelle, il peut accroître le débordement d'une sphère vers l'autre et notamment du travail dans le hors travail. Le principal sujet d'intrusion est l'usage du téléphone personnel et par conséquent les appels peuvent déborder hors des horaires de travail, en cas de non-transfert de ligne. Notons que les télétravailleurs font un usage de plusieurs outils personnels pour un quart à un tiers des télétravailleurs. Plusieurs problèmes se posent. Un premier est celui de la prise en charge financière du matériel nécessaire au travail par l'employeur. Un autre problème est celui de la protection des données lorsque plusieurs supports d'enregistrement sont utilisés (clé usb, ordinateur personnel, messagerie personnelle...).

En phase d'évaluation préalable au télétravail le manager doit mettre l'agent face aux difficultés susceptibles d'engendrer une intrusion du travail dans la vie privée, ainsi qu'aux risques d'empiétement de la famille sur le travail notamment en cas de présence de la famille aux horaires de travail pouvant induire des interruptions qui perturbent le travail. Il est probable que la flexibilité offerte par le télétravail réduise l'impact de tensions ou d'un stress mais peut aussi en introduire de nouveaux : difficulté à se concentrer, à ne pas s'éparpiller, sentiment de devoir envers la maison ou la famille... Face à un risque de brouillage, il est important que le télétravailleur à domicile définisse des règles au sein de sa famille (horaires de travail, appels passés durant le travail...). Plusieurs agents ont expliqué que l'arrivée des enfants de l'école ou les horaires des activités personnelles ou familiales bornaient la journée de travail. Nos résultats sont cohérents avec ceux de Metzger et Cléach (2004,p. 443), «pour résister, il faut avoir appris à se fixer des objectifs, mais également à s'imposer des bornes. Savoir «quand arrêter le travail » devient une compétence à part entière ». Des conjoints n'ont peut-être pas nécessairement envie d'être confrontés chez eux à l'intrusion de la sphère professionnelle (Mezger et Créach, 2004), surtout lorsqu'ils sont éloignés du milieu professionnel (cas des conjoints retraités). Ils peuvent par ailleurs contribuer à la limitation de cette confusion en rappelant les devoirs familiaux (goûter des enfants, devoirs, bains...). Comme l'indique Mezger et Créach (2004, p. 448), « le contrôle social dévolu à l'entreprise est transféré vers l'environnement familial, activité pour laquelle les intéressés ne sont ni légitimes, ni reconnus et encore moins rémunérés [...] Finalement une part importante des télétravailleurs parvient avec l'appui de leurs proches, à développer des 
stratégies d'endiguement leur permettant de limiter la confusion des genres entre sphères privée et publique ». "La famille a alors un rôle de garde-fou contre le surtravail et plus largement pour définir des frontières entre travail et hors-travail » (Klein et Ratier, 2012; p. 184). Ce décentrage du contrôle social contribue à importer au sein de la sphère familiale un risque jusqu'ici supporté par l'entreprise, ajoutant une responsabilité/compétence d'auto-organisation au télétravailleur.

L'effet intégrateur par nature du télétravail est limité par la durée maximum de deux jours de télétravail, qui conjuguée à la règle de la réversibilité, ont limité l'effet intégrateur de cette forme d'organisation dans la vie privée des personnes. En effet, les règles qui encadrent le télétravail limitent les changements qui pourraient survenir dans la sphère familiale. Si l'organisation familiale peut parfois reposer sur le télétravailleur à domicile jugé plus disponible, la réversibilité du télétravail limite ces changements à des arrangements, à de la souplesse (moins de temps passé par les enfants à la garderie par exemple). Si ce n'était pas le cas, l'organisation familiale serait encore plus bouleversée et devrait s'adapter en cas de réversibilité. En revanche, le télétravail permet de mieux gérer son temps et de passer plus de temps en famille. Un directeur adjoint a fait le choix de télétravailler pour des raisons familiales. « Je me suis rendu compte que j'étais rarement avec les enfants. Je suis présent pour des moments clés et essentiels pour la vie de mes enfants ». Néanmoins, il sent une difficulté à maintenir cette journée de télétravail par semaine. Ces résultats font écho aux travaux sur la relative stabilité des frontières des vies personnelle et professionnelle (Dumas, 2008b).

Troisièmement, les résultats illustrent la capacité d'adaptation et d'autonomie des agents. En premier lieu, les télétravailleurs non-cadres ont su s'organiser et font preuve de capacités d'auto-organisation sans être sous le contrôle de leur chef. Globalement les télétravailleurs ont pris conscience de la nécessité d'être beaucoup plus organisés qu'avant : planifier ce qui peut être fait à distance ou pas, planifier les jours de contact avec le public, anticiper son programme de travail, garder de la rigueur dans cette organisation, faire un suivi d'activité plus régulier et plus formalisé, assurer la visibilité de ses horaires de travail, recadrer et formaliser la part du poste nécessitant des rencontres avec les collègues. Un agent dit avoir trouvé la valeur plaisir dans le télétravail. La qualité du travail du fait de l'épanouissement des agents est bénéfique pour la collectivité territoriale. D'autres recherches montrent que la participation aux domaines du hors-travail peut influencer favorablement la satisfaction dans l'emploi (Kirchmeyer, 1992; Dumas, 2008b). Une vie familiale intense, par exemple, a été fortement associée à la satisfaction dans l'emploi. Le processus par lequel la participation dans les domaines du hors-travail peut enrichir les ressources personnelles des salariés a été analysé. Le temps passé dans certains domaines du hors-travail peut augmenter l'offre de ressources disponibles pour le travail. Les personnes interrogées perçoivent cette participation comme enrichissante sur de nombreux plans : elle permet notamment de développer des compétences utiles pour le travail. En second lieu, le télétravail apporte une souplesse face à des problèmes externes. Le télétravail peut se révéler une solution intéressante notamment lors d'intempéries. En outre le changement de lieu de travail en cas de télétravail en télécentre est propice à des rencontres riches qu'ils n'avaient pas anticipées : par exemple des rencontres avec des élus qui ont des bureaux dans le même bâtiment. Enfin le télétravail répond aux attentes d'Administration en tant que vecteur de changements culturel et managérial. Des agents en télétravail ont accepté des modifications, en particulier, du mode de contrôle de leurs activités (cas des informaticiens) 
qu'ils jugent justifiées par le télétravail, par son développement, ainsi que par des difficultés d'ordre managérial induites. La logique semble gagnant-gagnant, même si certains cadres estiment que l'avantage est largement du côté des télétravailleurs.

\section{Conclusion}

L'étude par entretiens et questionnaires montre que le télétravail est favorable à la conciliation des sphères travail et hors travail. Il peut toutefois favoriser un brouillage des frontières avec notamment l'empiétement d'une sphère par l'autre, l'addiction ou le travail sans fin.

Les intrusions sont vécues comme des violations de la frontière. Les outils de communication en particulier le téléphone est vécu comme tel. Les agents auraient paradoxalement préféré être équipés d'un téléphone portable. Un autre élément d'intégration est l'utilisation d'outils informatiques personnels à des fins professionnelles.

Toutefois ce phénomène de brouillage est limité puisque le télétravail étudié est une pratique d'intégration du travail dans la vie des personnes limitée du fait de la durée et des règles définies par les cadres (segmentation). Les personnes segmentent en se fixant des règles établies dans le cadre familial (arrivée des enfants) ou lié à une activité personnelle. Les horaires de travail sont fortement déterminés par l'organisation personnelle et familiale et non plus organisationnelle.

L'étude montre par ailleurs que les agents ont fait preuve de capacités et de compétences à s'organiser et à gagner en autonomie.

L'apport de ce travail est de fournir des résultats qualitatifs et quantitatifs auprès non seulement de télétravailleurs mais de personnes de leur environnement de travail, qui sont les managers et les collègues. S'il y a une tendance naturelle à valoriser dans le discours une pratique appréciée à laquelle ils sont attachés, le croisement des réponses des télétravailleurs, des collègues et des encadrants et leur comparaison permet de contrôler ce biais. Par ailleurs chaque thème comporte des questions affirmatives et négatives avec en réponse un large choix de propositions.

Nous regrettons que le nombre de répondants ne soit pas supérieur, notamment pour les encadrants et les collègues. Le nombre de réponses des télétravailleurs est très satisfaisant. Il semble que la question portant sur les effets négatifs du télétravail ait bloqué certains répondants et explique certaines réponses partielles (questionnaires exclus).

Le télétravail a permis aux salariés de gagner en autonomie d'organisation. Il sera intéressant d'évaluer l'impact du télétravail sur quelques pratiques. Ainsi l'attachement au télétravail pourrait devenir un frein à la mobilité du fait des conditions de mise en œuvre différentes selon les services et du fait de l'interruption du télétravail, bien que temporaire, suite à un changement de service. Par ailleurs, la réforme des rythmes scolaires et notamment l'école le mercredi matin pourra être un facteur de développement du télétravail. Les parents de jeunes enfants pourront souhaiter télétravailler le matin et prendre un temps partiel pour passer le mercredi après-midi avec les enfants, économisant ainsi un déplacement pour une demi-journée de travail. 


\section{Bibliographie}

Baines S., Gelder U., 2003. What is Family Friendly about the Workplace in the Home? The Case of Self Employed Parents and their Children. New Technology, Work and Employment 18 (3), 223-231.

Bourhis A., Mekkaoui R., 2010. Beyond Work-Family Balance: Are Family-Friendly more Attractive? Relations Industrielles, Industrial Relations 65 (1), 98-117.

Brey P., 1999. Worker Autonomy and the Drama of Digital Networks in Organizations. Journal of Business Ethics 22, 15-25.

Christensen K.E., 1987. Impacts of Computer-Mediated Home Based Work on Women and their Families. Office, Technology and People 3, 211-230.

Clark S.C., 2000. Work/family Border Theory: A new Theory of Work/Family Balance. Human Relations 53 (6), 747-770.

Centre d'analyse stratégique, 2013. Quel est l'impact des TIC sur les conditions de travail dans la fonction publique ? La note d'analyse Travail Emploi 318.

De Mazenod X., 2011. Le guide du télétravail. Rapport pour le projet e-Incorporate pour Soho-Solo de la CCI du Gers.

Dumas M., 2008a. Vie personnelle et vie professionnelle : vers un nouvel équilibre dans l'entreprise? Éditions EMS, Paris.

Dumas M., 2008b. Conflit et enrichissement travail-famille et implication. Revue de Gestion des Ressources Humaines 67, 23-37

Dumas M., Ruiller C., 2014. Le télétravail : les risques d'un outil de gestion des frontières entre vie personnelle et vie professionnelle ? Revue Management et Avenir 74, 71-95

Edley P.P., 2001. Technology, Employed Mothers, and Corporate Colonization of the Lifeworld: A Gendered Paradoxe of Work and Family Balance. Women \& Language 24 (2), 28-35

Fehrenbach J., Granel F., Dufort D., Klein T., Loyer J.L., 2009. Le développement du télétravail dans la société numérique de demain. Rapports et Documents, Centre d'Analyse Stratégique.

Felstead A., Jewson N., 2000. In Work, at Home. Towards an Understanding of Home Working. Routledge, London.

Fernandez V., Guillot C., Marrauld L., 2014. Télétravail et travail à distance équipé. Revue Française de Gestion 238, 101-118.

Gajendran R.S., Harrison D.A., 2007. The Good, the Bad and the Unknown about Telecommuting: Meta-Analysis and Psychological Mediators and Individual Consequences. Journal of Applied Psychology 92, 1524-1541.

Golden T., Veiga J., 2005. The Role of Virtual Work in Understanding the Impact of Supervisory Relationships. Academy of Management Best Conference Paper.

Golden T.D., Veiga J.F., Simsek Z., 2005. Telecommuting's Differential Impact on Work-Family Conflict: Is there no Place like Home? Journal of Applied Psychology 91 (6), 1340-1350.

Haddon L., Lewis A., 1994. The Experience of Teleworking: an Annotated Review. International. Journal of Human Resource Management 5, 193-223.

Hill E.J., Hawkins A.J., Ferris M.S., Weitzman M., 2001. Finding an Extra Day a Week: The Positive Influence of Perceived Job Flexibility on Work and Family Life Balance. Family Relations 50 (1), 49-58.

Karasek R.A., 1979. Job Demands, Job Decision Latitude and Mental Strain: Implications for Job Redesign. Administrative Sciences Quarterly 24, 285-308.

Kirchmeyer C., 1992. Nonwork Participation and Work Attitudes: A test of Scarcity vs Expansion Models of Personal Resources. Human Relations 45 (8), 775-795

Klein T., Ratier D., 2012. L'impact des TIC sur les conditions de travail, Centre d'Analyse Stratégique. La Documentation française, Paris.

Kossek E.E., Lautsch B.A., Eaton S.C., 2006. Telecommuting, Control, and Boundary Management: Corelates and Policy used and Practice, Job Control, and Work-Family Effectiveness. Journal of Vocational Behavioral 68, 347-367. 
Kowalski K.B., Swanson J.A., 2005. Critical Success Factors in Developing Telework Programs. Benchmarking: An International Journal 12, 236-249.

Kreiner G.E., 2006. Consequences of Work-Home Segmentation or Integration: A Person-Environment Fit Perspective. Journal of Organizational Behavior 27, 485-507.

Kreiner G.E., Hollensbe E.C., Sheep M.L., 2009. Balancing Borders and Bridges: Negotiating the Work-Home Interface via Boundary Work Tactics. Academy of Management Journal 52 (4), 704-730.

Lebreton C., 2013. Les territoires numériques de la France de demain. Rapport à la ministre de l'égalité des Territoires et du Logement, avec le concours de l'Assemblée des Départements de France et du Commissariat Général à la Stratégie et de la Prospective.

Lefevre C., Pailhé A., Sollaz A., 2007. Comment les employeurs aident-ils leurs salariés à concilier travailfamille? Populations et Société 440.

Madsen S.R., 2003. The Effects of Home-Based Teleworking on Work-Family Conflict. Human Resource Development Quarterly 14 (1), 35

Mann S., Holdsworth L., 2003. The Psychological Impact of Teleworking: Stress, Emotions and Health. New Technology, Work and Employment 18 (3), 196-211.

Matthews R.A., Bernes-Farrek J.L., Bulger C.A., 2010. Advancing Measurement of Work and Family Domain Boundary Characteristics. Journal of Vocational Behavior 77, 447-460.

Metzger J.-L., Cléach O., 2004. Le télétravail des cadres : entre suractivité et apprentissage de nouvelles temporalités. Sociologie du travail 46, 433-450.

Morel à l'Huissier P., Turbé-Suetens N., 2012. Le Télétravail en France. Village Mondial, Pearson, Paris.

Mustafa M., 2012. Work-Life Balance or Work-Life Ambivalence? Managing Flexibility amongst Self-Employed Teleworkers. International Journal of Scientific \& Engineering Research 3 (8).

Nippert-Eng C.E., 1996. Home and Work: Negotiating Boundaries Through Everyday Life. University of Chicago, Chicago.

Othman N., Yusef S.A.M., Osman W.R.S., 2009. A Conflict between Professional vs Domestic Life? Understanding the use of ICT in Teleworking for Balance in Work and Family Units. Computer and Information Sciences 2 (2).

Rau B.L., Hyland M.A., 2002. Role Conflict and Flexible Work Arrangements: The Effects on Applicant Attraction. Personnel Psychology 55 (1), 111-136.

Rey C., SitnikoffF., 2006. Télétravail à domicile et nouveaux rapports au travail. Revue Interventions Économiques 34, 1-16.

Schweitzer L, Duxbury L., (2006). Benchmarking the Use of Telework Arrangements in Canada. Canadian Journal of Administrative Sciences 23 (2), 105-117.

Tremblay D.G., 2002. Balancing Work and Family with Telework? Organizational Issues and Challenges for Women and Managers. Women in Management 17 (3/4), 157-170

Tremblay D.G., 2003. Telework: A new Model of Gendered Segmentation? Results from a Study in Canada. Canadian Journal of Communication 28 (4), 461-478.

Tremblay D.-G., Chevrier C., Di Loreto M., 2006. Le télétravail à domicile : Meilleure conciliation emploifamille ou source d'envahissement de la vie privée ? Revue Interventions Économiques 34, 1-16 


\section{Annexe 1 : Récapitulatif des personnes interviewées}

25 personnes interrogées ( 7 directeurs ou directeurs adjoints ; 4 responsables ou chefs de service; 14 agents), dont 20 télétravailleurs (4 directeurs ou directeurs adjoints ; 2 responsables ou chefs de service; 14 agents), soit 14 femmes et 6 hommes. Parmi les 20 télétravailleurs, 5 ont expérimenté le télétravail ( 2 femmes et 3 hommes), c'est-à-dire qu'ils télétravaillent depuis octobre 2009. Nous avons également rencontré 10 managers de télétravailleurs (directeurs ou chefs de service) ( 5 femmes et 5 hommes) dont 5 télétravaillent ( 3 femmes et 2 hommes).

\section{Par direction et métiers :}

La Direction des Ressources Humaines : 6 agents (drh; drh adjoint; chef de service formation accompagnement ; adjoint service formation ; Études et statistiques des effectifs ; assistante emploi)

La Direction des Services Informatiques : 4 agents (directeur; chef de service; 2 chefs de projet)

Le social : 8 agents (DEF : directeur adjoint ; CDEF assistante administrative ; assistante sociale) (TAS : déléguée thématique à l'insertion et action sociale), (DGAS : service ressources log pour les TAS) (DILE : directeur, chef de service; responsable de cellule RSA)

La direction des collèges : 2 agents (directeur; directeur adjoint)

Direction Générale : 3 agents (DAPI assistante; assistantes DGAR)

Autre direction : 2 agents (direction de l'aménagement de l'eau et du logement; Les routes)

\section{Annexe 2 : Les thèmes des questionnaires}

Télétravailleurs : 26 questions portant sur l'organisation du télétravail (temps, rythme, lieu, respect des jours, différents changements) ; les impacts sur l'organisation, le management, la relation aux usagers, partenaires, collègues (effets positifs, négatifs du télétravail, communication en situation de télétravail, motifs de retour au bureau); la gestion des temps et des lieux (transport, télécentre/domicile, conciliation vie professionnelle et vie personnelle, effets positifs pour la famille, adaptation personnelle de son temps de travail, adaptation du télétravail à l'activité/missions du service; image du télétravailleur).

Encadrants : 10 questions portant sur les impacts sur l'organisation, le management, la relation au travail (approche managériale, organisation du service, communication avec les télétravailleurs, effets positifs, négatifs); regard de l'encadrant sur le télétravail dans l'institution (risques à l'exercice du télétravail, niveau de satisfaction global ressenti par l'encadrant, les télétravailleurs, les collègues des télétravailleurs).

Collègues des télétravailleurs : 12 questions portant sur les impacts sur l'organisation, le management, la relation aux autres (types d'impacts et appréciation, communication avec télétravailleurs); vous et le télétravail (distance domicile travail, télétravail et activité/missions, intérêt pour le télétravail, effets positifs, négatifs du télétravail, image du télétravailleur). 


\section{Annexe 3 : Description des échantillons de répondants (questionnaires exploités)}

Télétravailleurs (105) : 43 répondants appartiennent à la catégorie A (41\%) et 43 à la catégorie B $(41 \%)$ et 19 à la catégorie C (18\%). L'âge moyen est de 45 ans. Les femmes représentent près des trois quarts des répondants (78 femmes et 27 hommes).

Encadrants de télétravailleurs (35) : 18 hommes et 17 femmes ; Excepté deux personnes de catégorie $\mathrm{B}$, tous appartiennent à la catégorie $\mathrm{A}$. La moyenne d'âge est de 50 ans. Cinq encadrants télétravaillent.

Collègues de télétravailleurs (51) : Leur âge moyen est de 44 ans et sont en grande majorité des femmes $(72,5 \%)$. Près de $47 \%$ (24) sont de catégorie B et près de $15 \%$ de catégorie C (8). Les catégories A représentent 23,5\% des répondants (12).

\section{Annexe 4 : Télétravail et séparation entre vie professionnelle et votre vie professionnelle}

\begin{tabular}{|l|c|c|c|c|}
\hline $\begin{array}{l}\text { Le télétravail pose-t-il des problèmes } \\
\text { de séparation entre vie professionnelle } \\
\text { et votre vie professionnelle }\end{array}$ & Pas du tout & Plutôt non & Plutôt oui & Tout à fait \\
\hline Sollicitation par la famille & $73,3 \%$ & $23,8 \%$ & $2,9 \%$ & - \\
\hline Horaires tardifs & $42,9 \%$ & $40,0 \%$ & $12,4 \%$ & $4,8 \%$ \\
\hline $\begin{array}{l}\text { Réponse aux messages } \\
\text { hors temps de travail }\end{array}$ & $42,9 \%$ & $31,4 \%$ & $21,9 \%$ & $3,8 \%$ \\
\hline $\begin{array}{l}\text { Appels téléphoniques } \\
\text { en dehors des horaires de travail }\end{array}$ & $46,7 \%$ & $40,0 \%$ & $11,4 \%$ & $1,9 \%$ \\
\hline $\begin{array}{l}\text { Appels téléphoniques } \\
\text { sur mon poste de téléphone fixe }\end{array}$ & $45,7 \%$ & $25,7 \%$ & $14,3 \%$ & $14,3 \%$ \\
\hline
\end{tabular}


Annexe 5 : Télétravail et vie personnelle et vie professionnelle

\begin{tabular}{|c|c|c|c|c|}
\hline $\begin{array}{l}\text { Cadres : le télétravail pose-t-il } \\
\text { des problèmes de séparation } \\
\text { entre vie professionnelle } \\
\text { et votre vie personnelle: }\end{array}$ & Pas du tout & Plutôt non & Plutôt oui & Tout à fait \\
\hline Sollicitation par la famille & $58,1 \%$ & $37,2 \%$ & $4,7 \%$ & - \\
\hline Horaires tardifs & $34,9 \%$ & $41,9 \%$ & $16,3 \%$ & $7,0 \%$ \\
\hline $\begin{array}{l}\text { Réponse aux messages } \\
\text { hors temps de travail }\end{array}$ & $25,6 \%$ & $39,5 \%$ & $32,6 \%$ & $2,3 \%$ \\
\hline $\begin{array}{l}\text { Appels téléphoniques } \\
\text { en dehors des horaires de travail }\end{array}$ & $27,9 \%$ & $58,1 \%$ & $14 \%$ & - \\
\hline $\begin{array}{l}\text { Appels téléphoniques } \\
\text { sur mon poste de téléphone fixe }\end{array}$ & $32,6 \%$ & $34,9 \%$ & $18,6 \%$ & $14,0 \%$ \\
\hline $\begin{array}{l}\text { Non-cadres : Le télétravail pose-t-il } \\
\text { des problèmes de séparation entre vie } \\
\text { professionnelle et votre vie profes- } \\
\text { sionnelle : }\end{array}$ & Pas du tout & Plutôt non & Plutôt oui & Tout à fait \\
\hline Sollicitation par la famille & $83,9 \%$ & $14,5 \%$ & $1,6 \%$ & \\
\hline Horaires tardifs & $48,4 \%$ & $38,7 \%$ & $9,7 \%$ & $3,2 \%$ \\
\hline $\begin{array}{l}\text { Réponse aux messages hors temps de } \\
\text { travail }\end{array}$ & $54,8 \%$ & $25,8 \%$ & $14,5 \%$ & $4,8 \%$ \\
\hline $\begin{array}{l}\text { Appels téléphoniques en dehors des } \\
\text { horaires de travail }\end{array}$ & $59,7 \%$ & $27,4 \%$ & $9,7 \%$ & $3,2 \%$ \\
\hline $\begin{array}{l}\text { Appels téléphoniques sur mon poste de } \\
\text { téléphone fixe }\end{array}$ & $54,8 \%$ & $19,4 \%$ & $11,3 \%$ & $14,5 \%$ \\
\hline
\end{tabular}


Annexe 6 : Télétravail et conciliation vie personnelle et vie professionnelle

\begin{tabular}{|l|c|c|c|c|c|}
\hline Le télétravail permet de : & $\begin{array}{c}\text { Total/ \% } \\
\text { concernés }\end{array}$ & $\begin{array}{c}\text { Hommes } \\
(\mathrm{n}=27)\end{array}$ & $\begin{array}{c}\text { Femmes } \\
(\mathrm{n}=78)\end{array}$ & $\begin{array}{c}\text { Cadres } \\
(\mathrm{n}=43)\end{array}$ & $\begin{array}{c}\text { Non- } \\
\text { cadres } \\
(\mathrm{n}=62)\end{array}$ \\
\hline plus de temps en famille & $\mathbf{8 4 , 4} \%$ & $84 \%$ & $95,1 \%$ & $86,8 \%$ & $82,7 \%$ \\
\hline $\begin{array}{l}\text { un partage des tâches domes- } \\
\text { tiques ou familiales } \\
\text { avec mon conjoint }\end{array}$ & $47,3 \%$ & $43,4 \%$ & $49 \%$ & $60 \%$ & $38,6 \%$ \\
\hline $\begin{array}{l}\text { d'avoir une (des) activités associa- } \\
\text { tives et citoyennes }\end{array}$ & $38,2 \% \%$ & $43,4 \%$ & $36,2 \%$ & $44,1 \%$ & $34 \%$ \\
\hline plus de temps pour soi & $\mathbf{7 0 , 3} \%$ & $73 \%$ & $69,3 \%$ & $67,4 \%$ & $72,4 \%$ \\
\hline $\begin{array}{l}\text { facilite le mode de garde } \\
\text { des enfants }\end{array}$ & $58,3 \%$ & $71,4 \%$ & $52,9 \%$ & $65 \%$ & $53,6 \%$ \\
\hline $\begin{array}{l}\text { faire moins de choses le week-end } \\
\text { (courses et tâches ménagères) }\end{array}$ & $48 \%$ & $36 \%$ & $52,1 \%$ & $52,5 \%$ & $44,6 \%$ \\
\hline
\end{tabular}

\section{Annexe 7 : Télétravail et vie de famille}

\begin{tabular}{|l|c|c|c|c|c|}
\hline $\begin{array}{l}\text { Le télétravail est bénéfique } \\
\text { pour la famille car il permet de : }\end{array}$ & $\begin{array}{c}\text { Total/ } \% \\
\text { concernés }\end{array}$ & $\begin{array}{c}\text { Hommes } \\
(\mathrm{n}=27)\end{array}$ & $\begin{array}{c}\text { Femmes } \\
(\mathrm{n}=78)\end{array}$ & $\begin{array}{c}\text { Cadres } \\
(\mathrm{n}=43)\end{array}$ & $\begin{array}{c}\text { Non- } \\
\text { cadres } \\
(\mathrm{n}=62)\end{array}$ \\
\hline $\begin{array}{l}\text { passer plus de temps } \\
\text { avec les enfants }\end{array}$ & $72 \%$ & $76,2 \%$ & $69,7 \%$ & $63,8 \%$ & $70,2 \%$ \\
\hline $\begin{array}{l}\text { passer plus de temps } \\
\text { avec le conjoint }\end{array}$ & $68,7 \%$ & $68,1 \%$ & $73 \%$ & $66,6 \%$ & $68 \%$ \\
\hline $\begin{array}{l}\text { participe à un partage } \\
\text { des tâches dans le couple }\end{array}$ & $54,4 \%$ & $45,4 \%$ & $43,8 \%$ & $58 \%$ & $35,4 \%$ \\
\hline $\begin{array}{l}\text { passer plus de temps } \\
\text { auprès des personnes âgées. }\end{array}$ & $33,3 \%$ & $21,4 \%$ & $37,5 \%$ & $34,8 \%$ & $32,2 \%$ \\
\hline
\end{tabular}

